Correspondence

Ming-Chung Chang

mcchang@mail.ncku.edu.tw

I-Cheng Tseng

icheng@mail.ncku.edu.tw

Received 20 December 2008

Revised 11 April 2009

Accepted 15 April 2009

\section{Ferrous iron-binding protein Omb of Salmonella enterica serovar Choleraesuis promotes resistance to hydrophobic antibiotics and contributes to its virulence}

\author{
Jer-Horng Su, ${ }^{1,2}$ Yin-Ching Chung, ${ }^{3}$ Hsin-Chun Lee, ${ }^{4}$ I-Cheng Tseng ${ }^{2}$ \\ and Ming-Chung Chang 5,6,7
}

${ }^{1}$ Department of Biotechnology, Chia-Nan University of Pharmacy and Science, 60, Erh-Jen Rd, Sec.1, Jen-Te, Tainan 717, Taiwan, ROC

${ }^{2}$ Department of Life Science, College of Bioscience and Biotechnology, National Cheng-Kung University, No. 1, University Road, Tainan 701, Taiwan, ROC

${ }^{3}$ Department of Medical Research, Chi Mei Medical Center, No. 901, Chung Hwa Road, Yong Kang City, Tainan 710, Taiwan, ROC

${ }^{4}$ Department of Internal Medicine, National Cheng-Kung University Hospital, No. 138, Sheng Li Road, Tainan 701, Taiwan, ROC

${ }^{5}$ Department of Biochemistry and Molecular Biology, College of Medicine, National Cheng-Kung University, Tainan 701, Taiwan, ROC

${ }^{6}$ Sustainable Environment Research Center, National Cheng-Kung University, Tainan 701, Taiwan, ROC

${ }^{7}$ Research Institute of Biotechnology, Hungkuang University, No. 34, Chung-Chie Rd, Sha Lu, Taichung County, Taiwan 43302, ROC

Salmonella enterica serovar Choleraesuis (SC) is an important enteric pathogen that causes serious systemic infections in swine and humans. To identify the genes required for resistance to antimicrobial peptides, we constructed a bank of SC transposon mutants and screened them for hypersensitivity to the cationic peptide polymyxin B. Here we report one isolated polymyxin Bsusceptible mutant that also exhibited increased sensitivity toward human neutrophil peptide alpha-defensin 1 (HNP-1) and hydrophobic antibiotics including erythromycin and novobiocin. The mutant had a mutation in an ORF identified as outer membrane $\beta$-barrel protein gene omb. The purified recombinant Omb protein was characterized as a ferrous iron-binding protein. The constructed omb isogenic mutant grew more slowly in iron-limiting conditions than the wild-type (WT) parent strain. In addition, compared with the WT strain, the omb mutant exhibited an increase in net negative charge upon the cell surface and was more easily killed by polymyxin B, HNP-1 and hydrophobic antibiotics. The omb gene was transcribed, regardless of the iron content within the growth medium, and the Omb protein appeared exclusively in the outer membrane fraction. Infection experiments demonstrated virulence attenuation when the mutant was administered orally or intraperitoneally to mice. This study indicates that Omb is a previously unrecognized ferrous iron-binding protein. In vivo, Omb may be involved in the acquisition of ferrous iron during the initial stages of $\mathrm{SC}$ infection and appears to be an important virulence factor for SC in mice.
Abbreviations: AP, antimicrobial peptide; 2,2-DPD, 2,2-dipyridyl; i.p., intraperitoneal; PG SK, phen green SK (dipotassium salt); SC, Salmonella enterica serovar Choleraesuis; WT, wild-type.

The GenBank accession number for the omb sequence of Salmonella enterica serovar Choleraesuis S280 is FJ178865.

\section{INTRODUCTION}

Salmonella enterica serovar Choleraesuis (SC) is a Gramnegative and facultative intracellular bacterium that causes serious systemic infections including typhoid disease, bacteraemia, pneumonia, septicaemia, enterocolitis, hepatitis, encephalitis and abortion in swine (Schwartz, 1999). 
Although SC is highly host-adapted, the organism is also notorious for its extreme invasiveness and pathogenic nature in humans, frequently causing septicaemic disease with only marginal involvement of the intestinal tract (Chiu et al., 2006, 2005). In Taiwan, SC is the second most common of all Salmonella serotypes isolated and shows the greatest ability to cause extraintestinal infections (Wang et al., 2006). Recently, an increasing percentage of highly invasive strains of SC exhibiting high-level resistance to antimicrobial agents have become a serious problem that hampers the effective treatment of diseases caused by this pathogen.

The ability of bacterial pathogens to cause disease in mammals is dependent upon their ability to overcome the host immune defence system. Antimicrobial peptides (APs) are one of the important weapons of this defence system. Though structurally highly diverse, nearly all APs share the features of a net cationic charge and have the capacity to kill a broad spectrum of micro-organisms. Electrostatic interactions between the positively charged APs and the negatively charged bacterial cell envelope are critical for bacterial killing. It is therefore not surprising that bacterial resistance to APs is linked to charge-based modifications at the cell surface, which result in decreased binding of the cationic APs and afford the organism relative protection. Examples include the addition of L-lysine to phosphatidylglycerol (Kristian et al., 2003), the addition of aminoarabinose to the lipopolysaccharide (LPS) lipid A moiety (Tamayo et al., 2005), and the incorporation of Dalanine into Gram-positive cell wall teichoic acids (Kristian et al., 2005).

In an attempt to understand the mechanisms involved in the natural resistance of clinical isolates of SC to APs, we isolated a polymyxin B-susceptible Tn5 mutant that also exhibited increased sensitivity toward human neutrophil peptide alpha-defensin 1 (HNP-1) and hydrophobic antibiotics, including erythromycin and novobiocin. This paper reports the cloning of the Tn5-inactivated gene from this mutant and the characterization of its gene product.

\section{METHODS}

Bacterial strains and plasmids. The bacterial strains and plasmids used for this study are listed in Table 1. SC strain S280 is a clinical isolate from the blood of a patient at the National Cheng-Kung University Hospital, Taiwan, ROC. All strains were routinely grown in minimal medium (12.8 $\mathrm{g} \mathrm{Na}_{2} \mathrm{HPO}_{4} .7 \mathrm{H}_{2} \mathrm{O} \mathrm{l} \mathrm{l}^{-1}, 3 \mathrm{~g} \mathrm{KH}_{2} \mathrm{PO}_{4} \mathrm{l}^{-1}$, $10 \mathrm{~g} \mathrm{NaCl}^{-1}, 1 \mathrm{~g} \mathrm{NH}_{4} \mathrm{Cl}^{-1}, 2 \mathrm{mM} \mathrm{MgSO}_{4}, 0.2 \mathrm{mM} \mathrm{CaCl}_{2}, 4 \mathrm{~g}$ glucose $1^{-1}$ ) or Luria-Bertani (LB) medium at $37{ }^{\circ} \mathrm{C}$, with aeration. Antibiotics were used as follows: $100 \mu \mathrm{g}$ ampicillin $\mathrm{ml}^{-1}, 100 \mu \mathrm{g}$ kanamycin $\mathrm{ml}^{-1}$ and $50 \mu \mathrm{g}$ tetracycline $\mathrm{ml}^{-1}$ for both Escherichia coli and SC. MilliQ (Millipore) water was used for all growth media and chemical solutions. All iron-deficient media were prepared in plastic containers and filter-sterilized, and cells were grown in polypropylene and polystyrene vessels (Knight et al., 2005).

Molecular techniques. Standard techniques were used to construct recombinant plasmids (Sambrook et al., 1989). DNA fragments used in cloning were extracted from agarose gels by use of the Qiaex II kit
(Qiagen). PCR was carried out according to the manufacturer's recommendations by use of the Taq DNA polymerase kit (GE Healthcare Biosciences). Nucleotide sequences were determined with an autosequencer (ABI Prism 373 DNA sequencer; Applied Biosystems).

Construction of transposon mutant bank. The transposon insertional library of SC strain S280 was generated by a published method (Hensel et al., 1995), with minor modifications. E. coli S17-1 $\lambda$ pir carrying a transposon plasmid (Hensel et al., 1995) was delivered to the tetracycline-resistant SC strain S280 by conjugation. The transconjugants were selected by growth with kanamycin and tetracycline. The resultant mutants were further screened by the polymyxin B sensitivity assay described below.

AP and hydrophobic antibiotic sensitivity assay. An AP and hydrophobic antibiotic sensitivity assay was performed as described elsewhere (Kristian et al., 2005), with minor modifications. In sterile 96-well microtitre plates, exponential-phase S280 or transposon mutant culture was adjusted to $10^{5}$ c.f.u. $\mathrm{ml}^{-1}$ in $100 \mu \mathrm{l}$ minimal medium containing polymyxin $\mathrm{B}\left(0-10 \mu \mathrm{g} \mathrm{ml}^{-1}\right)$ (Gibco BRL), HNP-1 (0-50 $\left.\mu \mathrm{g} \mathrm{ml}^{-1}\right)$ (Sigma), erythromycin $\left(0-50 \mu \mathrm{g} \mathrm{ml}{ }^{-1}\right)$ (Fluka), or novobiocin $\left(0-2 \mathrm{mg} \mathrm{ml}^{-1}\right)$ (Sigma), in serial dilutions. The MIC was defined as the lowest antimicrobial concentration yielding no detectable bacterial growth $\left(\mathrm{OD}_{600}\right.$ measurement). All experiments were done with duplicate samples on three independent occasions.

Colony and Southern hybridization. Colony hybridization and Southern hybridization were performed as described elsewhere (Sambrook et al., 1989). DNA probes were labelled with $\left[\alpha-{ }^{32} \mathrm{P}\right] \mathrm{dCTP}$ by use of a random priming kit (Megaprime DNA labelling system, GE Healthcare Biosciences), using either the PCR products or fragments excised from the recombinant plasmids as templates. The nylon membrane with DNA was prehybridized with hybridization buffer (ExpressHyb hybridization solution; Clontech Laboratories) for $30 \mathrm{~min}$ at $65{ }^{\circ} \mathrm{C}$, hybridized for $2 \mathrm{~h}$ at $65{ }^{\circ} \mathrm{C}$, washed, and visualized by autoradiography.

Analysis of transposon insertion sites. Chromosomal DNA from each Tn5 mutant was digested individually with BglII, EcoRI, KpnI, PstI and SalI (there are no recognition sites for these five restriction enzymes within the transposon). The presence of the Tn 5 mutant was screened for with an $\alpha^{-32} \mathrm{P}$-labelled kanamycin-resistance gene by Southern hybridization. The kanamycin probe was generated by excision from plasmid pUC4K to generate a $1.2 \mathrm{~kb}$ Sall fragment, which was then used as a template for the random priming kit.

Cloning of the SC omb gene. Chromosomal DNA from strain S280-1 was digested with EcoRI and inserted into identically digested pUC19. The ligation reaction mixtures were transformed into E. coli XL1Blue and then cells were selected for kanamycin resistance. Plasmid DNA was extracted, and the chromosomal DNA sequences flanking the transposon were obtained by DNA sequencing using primers P6 and P7 (Hensel et al., 1995). A genomic library of S280 was constructed in E. coli by using Sau3AI partially digested S280 DNA ligated into the BamHI site of pBR322, as described previously (Chang et al., 1993). The complete coding sequence of omb was cloned from the genomic library of the S280 strain by colony hybridization with an $\alpha-{ }^{32} \mathrm{P}$-labelled DNA fragment of the omb gene. One positive clone was selected for DNA sequencing.

Overexpression of Omb in E. coli. A $663 \mathrm{bp}$ fragment of the omb gene was amplified from plasmid pSC by PCR with primers F4 (5'CTGTCATGGTCGACCCCTCCACCGCGAAA-3') and R1 (5'TCTTCTCGAGCAGCGTTACCGTAGTGCGG-3'). PCR products were digested with $B a m \mathrm{HI}$ and $\mathrm{XhoI}$ and inserted into identically 
Table 1. Bacterial strains and plasmids used in this study

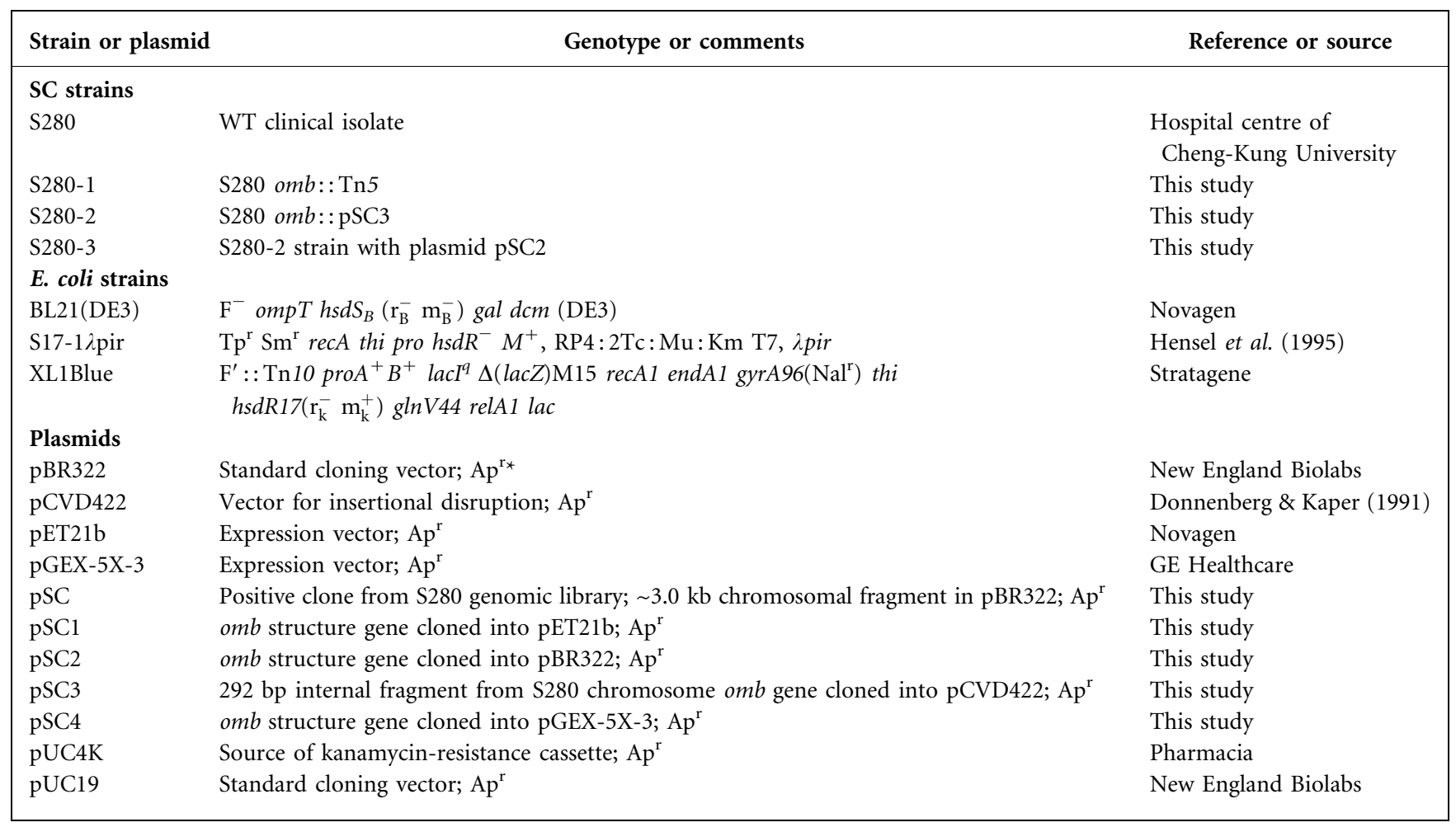

${ }^{\star} \mathrm{Ap}^{\mathrm{r}}$, ampicillin resistance.

digested pGEX-5X-3 (GE Healthcare) to generate plasmid pSC4. The GST-Omb fusion protein containing the amino acid region of Omb between Asp-34 and Leu-248 was expressed in E. coli BL21(DE3). The transformants were grown at $37{ }^{\circ} \mathrm{C}$ in LB medium containing $100 \mu \mathrm{g}$ ampicillin $\mathrm{ml}^{-1}$. When the culture reached $\mathrm{OD}_{600} 0.5$, IPTG was added to a final concentration of $1.0 \mathrm{mM}$. After a further $4 \mathrm{~h}$ of growth, the cultures were harvested by centrifugation at 4000 r.p.m. for $15 \mathrm{~min}$ at $4{ }^{\circ} \mathrm{C}$. The pellet was resuspended in PBS containing $0.05 \%$ Tween-20 (PBST) in $1 \%$ of the original volume and lysed by sonication on ice, and then centrifuged at 10000 r.p.m. for $20 \mathrm{~min}$ at $4{ }^{\circ} \mathrm{C}$. The supernatant was applied to glutathione-Sepharose $4 \mathrm{~B}$ beads (Pharmacia) and extensively washed with PBST. Factor Xa was injected into the column and the column was maintained at room temperature overnight. Using this method, the fusion protein was site-specifically cleaved and the recombinant Omb was eluted with $50 \mathrm{mM}$ Tris/ $\mathrm{HCl}$ ( $\mathrm{pH}$ 7.7) containing $100 \mathrm{mM} \mathrm{NaCl}$. The yield of the Omb by this method was estimated at about $4 \mathrm{mg}$ from 11 bacterial culture.

Iron-binding assay. An iron-binding assay was performed as described elsewhere (Petrat et al., 1999), with minor modifications. The fluorescent dye phen green SK (dipotassium salt) (PG SK) for iron detection was purchased from Invitrogen Molecular Probes. $\mathrm{Fe}^{2+}$ and $\mathrm{Fe}^{3+}$ stock solutions were always freshly prepared from ferrous ammonium sulfate and ferric chloride, respectively, and were dissolved in a simple buffered solution containing $2 \mathrm{mM}$ ascorbate (freshly prepared) in imidazole buffer $(10 \mathrm{mM}, \mathrm{pH}$ 7.2). Spectra of free PG SK were scanned with a spectrofluorometer (Firma Jobin Yvon, type JY3) at a speed of $1500 \mathrm{~nm} \mathrm{~min}{ }^{-1}$. For the fluorescence measurements, a simple buffered solution of purified Omb and iron was incubated at room temperature for $1 \mathrm{~h}$. Then, aliquots $(1 \mathrm{ml})$ of the solution were transferred into the cuvette of the spectrofluorometer, and PG SK (final concentration $4 \mu \mathrm{M}$ ) was added and mixed with the solution to detect the fluorescence. After the baseline fluorescence had been recorded, a protein-free sample or an iron-free sample was prepared in the same way and used as a control. All experiments were repeated at least three times.

Preparation of polyclonal antisera. A $665 \mathrm{bp}$ fragment of the omb gene was amplified from plasmid pSC by PCR with primers F1 ( $5^{\prime}$ TACTCATATGGATGATCCCTCCACCGCGA-3') and R1 (5'TCTTCTCGAGCAGCGTTACCGTAGTGCGG-3'). PCR products were digested with $\mathrm{NdeI}$ and $\mathrm{XhoI}$ and inserted into identically digested pET21b (Novagen) to generate plasmid pSC1. His ${ }_{6}$-tagged Omb containing the amino acid region of Omb between Met-33 and Leu-248 was expressed in E. coli BL21(DE3) and purified under denaturing conditions by using a nickel affinity column, according to the manufacturer's instructions (Novagen). Purified His $_{6}$-tagged Omb at $100 \mu \mathrm{g}$ ( $\mathrm{ml}$ saline $)^{-1}$ was mixed with $1 \mathrm{ml}$ Freund's incomplete adjuvant. This mixture was then injected subcutaneously into an Elite New Zealand White rabbit. Two booster doses were administered at 2-week intervals, and the antiserum was collected after 6 weeks. The antiserum was purified through a protein A column according to the manufacturer's instructions (GE Healthcare Biosciences).

Western blot analysis. Proteins separated by SDS-12\% PAGE were electroblotted onto a nitrocellulose membrane (GE Healthcare Biosciences) and then incubated with a $\mathrm{His}_{6}$-tagged Omb-specific rabbit polyclonal antibody as the primary antibody. The secondary antibody was a 1:15000 dilution of goat anti-rabbit IgG conjugated to horseradish peroxidase (Sigma). The proteins were visualized by 
chemiluminescence according to the manufacturer's protocol (ECL Western Blotting Detection Reagent; GE Healthcare Biosciences).

Cellular fractionation. Extracellular, periplasmic and cytoplasmic cell fractions were isolated as described elsewhere (Cornelis et al., 1982). The sarcosine-insoluble outer membrane fraction of S280 was prepared as described elsewhere (Doig \& Trust, 1994), with minor modifications. The cell pellets were disrupted by passage through a French pressure cell [20000 p.s.i. (138000 kPa)]. After removal of unbroken cells, the total membrane (pellet) and cytoplasmic (supernatant) fractions were collected by centrifugation $(40000 \mathrm{~g}$ $\left.30 \mathrm{~min}, 4{ }^{\circ} \mathrm{C}\right)$, resuspended in $20 \mathrm{mM}$ Tris $/ \mathrm{HCl}(\mathrm{pH} 7.5)$ containing $2.0 \%(\mathrm{w} / \mathrm{v})$ sodium lauryl sarcosine, and incubated at room temperature for $30 \mathrm{~min}$. Outer membrane proteins were collected by centrifugation $\left(40000 \mathrm{~g}, 30 \mathrm{~min}, 4{ }^{\circ} \mathrm{C}\right)$ and washed three times with distilled water.

Effect of iron on the growth of the SC omb mutant. Strains were grown at $37^{\circ} \mathrm{C}$ in LB broth and harvested at their mid-exponential phase of growth. Bacteria were centrifuged for $5 \mathrm{~min}$ at $3000 \mathrm{~g}$, washed twice with minimal medium, and resuspended in minimal medium to $\mathrm{OD}_{600} 0.1$. Bacterial suspensions were added at $1: 100$ to minimal medium containing different concentrations of the iron chelator 2,2'-dipyridyl (2,2-DPD) (Sigma Chemical) $\left(5 \times 10^{-4}\right.$ and $\left.1 \times 10^{-3} \mu \mathrm{M}\right)$ and incubated at $37^{\circ} \mathrm{C}$. The growth rate of the cells was monitored by $\mathrm{OD}_{600}$ measurements. Samples were shaken gently, and aliquots removed at specified times were assayed for bacterial counts by plating out serial dilutions onto LB plates. The doubling times $(g)$ for the number of bacteria in iron-limited medium were calculated with the following equation:

$g=\frac{t}{n}=\frac{0.301 t}{\left(\log N_{t}-\log N_{0}\right)}$

where $N_{0}$ is the initial population number, $N_{t}$ is the population number at time $t$, and $n$ is the number of generations in time $t$.

Cytochrome $\mathbf{c}$ binding assay. A cytochrome $c$ binding assay was performed as described previously (Kristian et al., 2005). Bacteria were grown to early exponential phase, washed twice with MOPS buffer $(20 \mathrm{mM}, \mathrm{pH} 7)$, adjusted to a final $\mathrm{OD}_{600}$ of 7 in MOPS buffer plus $0.5 \mathrm{mg}$ cytochrome $c \mathrm{ml}^{-1}$ (Sigma-Aldrich), and incubated at room temperature. A plate counting assay was also performed to verify the numbers of viable cells. As a control, $0.5 \mathrm{mg}$ cytochrome $c \mathrm{ml}^{-1}$ was incubated in MOPS buffer under the same conditions but without bacteria. After $10 \mathrm{~min}$, bacteria were removed by centrifugation $(21000 \mathrm{~g}, 3 \mathrm{~min})$ and the cytochrome $c$ content of the supernatants was quantified photometrically at $530 \mathrm{~nm}$, the absorption maximum of the prosthetic group.

Construction of the omb mutant. A 292 bp internal fragment of the $o m b$ gene from pSC was generated by PCR with primers F2 ( $5^{\prime}$ CTGGCATGCACAACGTACCGCCT-3') and R2 (5'-GGTGTCGACCGTATGTTCGTCATA-3'). The PCR product was digested with SphI and SalI and inserted into identically digested pCVD422 (Donnenberg \& Kaper, 1991). The resultant plasmid, pSC3, was transformed into E. coli S17-1 $\lambda$ pir and subsequently transferred into SC strain S280 via conjugation according to a published method (Hensel et al., 1995). Transconjugants were selected by using ampicillin and tetracycline. The resultant strain was further confirmed by PCR and Southern blot analysis using the omb probe.

Complementation analysis. A $1126 \mathrm{bp}$ fragment of the omb gene was amplified from pSC by PCR with the primers F3 (5'-TGATGGATCCGCTATCGAGAGAG-3') and R3 (5'-ACCGGCATGCGTAACGTAACCCG-3'). The PCR product was digested with BamHI and SphI and inserted into identically digested pBR322. The resultant plasmid, pSC2, was introduced into S280-2 by using a published method (Beliavskaia et al., 2000). Transformants were selected by using ampicillin. The resultant strain was further verified by plasmid extraction and PCR.

Virulence assay. BALB/c mice (6-8 weeks old) purchased from the animal centre of the College of Medicine at National Cheng-Kung University were challenged by intraperitoneal (i.p.) injection or by feeding with a bacterial suspension. In feeding experiments, mice were starved for $6 \mathrm{~h}$ to clear the contents of the stomach before the oral challenge. A $0.1 \mathrm{ml}$ volume of $100 \mathrm{mM} \mathrm{NaHCO}$ was administered intragastrically to neutralize the stomach contents. A group of eight mice were challenged orally with $0.2 \mathrm{ml}$ per mouse of a 10-fold serially diluted (in PBS) bacterial suspension by using a round-tipped stainless steel feeding needle, and mortality was recorded at 3-4 weeks post-infection. Each experiment was performed at least twice with similar results. The $\mathrm{LD}_{50}$ for each strain was calculated by the method of Reed \& Muench (1938).

\section{RESULTS}

\section{Isolation and characterization of polymyxin B-sensitive mutant S280-1}

To identify the genes required for resistance to APs, we constructed a bank of Tn 5 mutants of a clinical strain of SC S280, and screened them for susceptibility to APs using AP sensitivity assays. One polymyxin B-sensitive clone, S280-1, was isolated and further investigated. Compared with the wild-type (WT) strain, this mutant not only exhibited clearly decreased MICs for polymyxin B and HNP-1, but also showed significantly enhanced susceptibility to hydrophobic antibiotics including erythromycin and novobiocin (Table 2). However, the sensitivity of S280-1 to other antibiotics, including streptomycin, tetracycline, ampicillin, nalidixic acid and chloramphenicol, remained unchanged (data not shown). The S280-1 mutant had the same colony morphology as the WT strain, and both grew at similar rates in LB medium or minimal medium (data not shown), suggesting that S280-1 was not an auxotrophic mutant.

Southern blot analysis with the kanamycin gene as the probe revealed that the S280-1 mutant contained a transposon insertion site (data not shown). The DNA sequences immediately flanking the Tn5 integration sites in the S280-1 mutant were obtained as described in Methods. The resultant plasmid was sequenced, and through sequence analysis, 630 bp of S280-1 chromosomal DNA beyond the transposon was cloned.

\section{Characterization of the genetic loci}

For isolation of the complete coding sequence of the Tn5interrupted gene, a genomic library of strain S280 DNA was subjected to colony hybridization, and a plasmid, pSC, obtained from a probe-reactive clone was selected for analysis. Three complete and one partial ORFs were identified. The DNA sequence of the first complete ORF (Fig. 1), where the mini-Tn5 was inserted in strain S280-1, 
Table 2. MICs for the APs and hydrophobic antibiotics used in this study

\begin{tabular}{|c|c|c|c|c|}
\hline \multirow[t]{3}{*}{ SC strain } & \multicolumn{4}{|c|}{$\operatorname{MIC}\left(\mu \mathrm{g} \mathrm{ml}^{-1}\right)$} \\
\hline & \multicolumn{2}{|c|}{ APs } & \multicolumn{2}{|c|}{ Hydrophobic antibiotics } \\
\hline & Polymyxin B & HNP-1 & Erythromycin & Novobiocin \\
\hline S280 (WT) & $0.458 \pm 0.014$ & $3.17 \pm 0.06$ & $3.33 \pm 0.06$ & $168 \pm 4$ \\
\hline S280-1 (Tn5 mutant) & $0.150 \pm 0.008^{*}$ & $0.67 \pm 0.03^{\star}$ & $0.83 \pm 0.03^{*}$ & $42 \pm 2^{*}$ \\
\hline S280-2 (isogenic mutant) & $0.167 \pm 0.006^{*}$ & $0.73 \pm 0.03^{\star}$ & $0.79 \pm 0.03^{*}$ & $38 \pm 2^{*}$ \\
\hline $\mathrm{S} 280-3(\mathrm{~S} 280-2+\mathrm{pSC} 2)$ & $0.471 \pm 0.011$ & $3.38 \pm 0.09$ & $3.58 \pm 0.06$ & $175 \pm 7$ \\
\hline
\end{tabular}

*Significantly lower MIC of AP/hydrophobic antibiotic for the mutant strain compared with the WT strain S280 (twotailed $t$ test, $P<0.05)$.

was designated omb (outer membrane $\beta$-barrel protein gene). The omb gene consists of a single transcriptional unit in which the coding sequence encodes a protein of 252 aa with a molecular mass of $27.8 \mathrm{kDa}$. The first 23 aa of the deduced Omb sequence have the characteristics of a typical bacterial signal sequence, followed by a typical AlaX-Ala signal peptidase cleavage site (von Heijne, 1983) between Ala-23 and Ala-24. Cleavage of the signal sequence would give a mature protein with a molecular mass of $25 \mathrm{kDa}$, which is in reasonable agreement with the size derived from SDS-PAGE (Fig. 2). The putative amino acid sequence of intact Omb shared $99-100 \%$ sequence identity with the putative outer membrane proteins of Salmonella typhimurium LT2 and CT18, and Salmonella paratyphi ATCC9150, as well as having high shared degrees of identity $(>54 \%)$ with the hypothetically extracytoplasmic proteins of several enteropathogenic E. coli strains and other pathogens, including Shigella spp., Yersinia spp., Klebsiella pneumoniae, Erwinia tasmaniensis and Serratia proteamaculans (data not shown). Only one of the aforementioned high-identity proteins has been characterized, the E. coli b1722 protein (Eb1722). Eb1722 has been classified as an outer membrane receptor (OMR) family protein by the $\beta$-barrel finder (BBF) program, although its amino acid sequence exhibits low similarity to known OMR family proteins (Zhai \& Saier, 2002). Since Eb1722 shares $88 \%$ sequence identity with Omb, it seems reasonable to propose that $\mathrm{Omb}$ is a member of the OMR family of proteins.

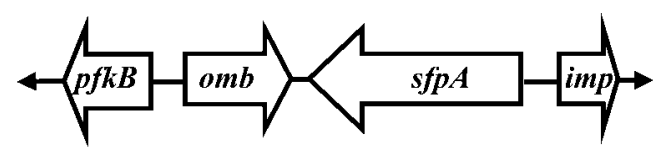

Fig. 1. Chromosomal organization of omb and the flanking region in SC S280. Arrows represent the direction of transcription and the ORFs of omb, sfpA and imp.
The second ORF was heterologously positioned $48 \mathrm{bp}$ downstream of omb and was predicted to encode a 319 residue protein with a molecular mass of $\sim 34.8 \mathrm{kDa}$. Its amino acid sequence shared $71 \%$ sequence identity with systemic factor protein A (SfpA) from Yersinia enterocolitica (Mildiner-Earley \& Miller, 2006), and was therefore designated $s f p A$ of SC. SfpA from Y. enterocolitica is a porin, located in the membrane, and is necessary for sustaining colonization of mesenteric lymph nodes; SfpA is conserved in other pathogenic bacteria that are involved in systemic disease (Mildiner-Earley \& Miller, 2006). The third ORF was heterologously positioned $142 \mathrm{bp}$ upstream of $s f p A$ and was predicted to encode a 104 residue protein with a molecular mass of $\sim 12.3 \mathrm{kDa}$. This amino acid sequence shared a high degree of sequence identity (96\%) with that of the putative inner membrane protein STM1329 [accession no. NP_460295 (gi: 16764680)] from S. typhimurium LT2, and was thus designated imp (putative inner membrane protein gene) from SC. The incomplete ORF was heterologously positioned 289 bp upstream of $o m b$ and potentially encoded the N-terminal 198 aa of a protein that shared significant homology (98\%) to 6-phosphofructokinase II from E. coli (Daldal, 1984) and was therefore designated $p f k B$ from SC. The genetic organization and the transcriptional direction of the three genes, omb, sfpA and imp of SC strain S280, strongly suggest that the mutant S280-1 phenotype is attributable only to the omb insertional mutation, since no polar effects on downstream genes should be expected. Recently, the complete genome sequence of SC strain SCB67 has become publicly available (http://www.ncbi.nlm. nih.gov/genomes/lproks.cgi?view=1). A comparison of the sequences of strain $\mathrm{S} 280 \mathrm{omb}$ and $s \mathrm{fp} A$ with those of strain $\mathrm{SC}-\mathrm{B} 67$ revealed that the respective sequences are the same at the nucleotide and amino acid levels. However, imp of strain S280 is an ORF encoding 104 aa of a protein, while the imp-related gene of SC-B67 has been designated a pseudogene (SCPS16) (http://www.ncbi.nlm.nih.gov/genomes/lproks.cgi?view=1) which encodes $82 \mathrm{aa}$, and is $100 \%$ identical with the N-terminal 82 aa sequence of S280 Imp. Nevertheless, the ORF of S280 imp is extended by an additional 22 aa at the $\mathrm{C}$ terminus. 


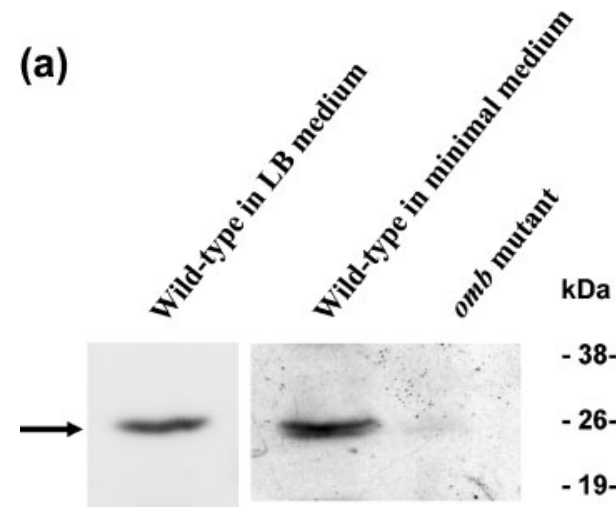

(b)

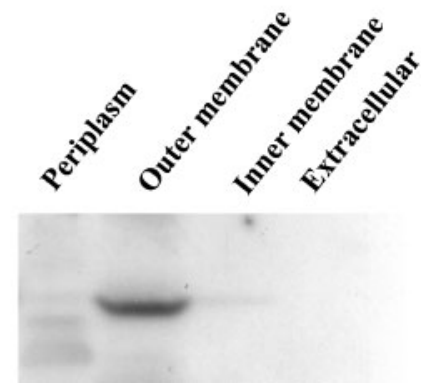

Fig. 2. Western blot analysis using polyclonal antibodies against the Omb protein. (a) Confirmation of the SC omb mutant by Western blotting. (b) Polypeptides from the various fractions of the WT strain $\mathrm{S} 280$. Proteins $(40 \mu \mathrm{g})$ were isolated from the WT and omb mutant strains, separated by electrophoresis, and transferred to a nitrocellulose membrane. Immunodetection of Omb was performed with a rabbit polyclonal anti-Omb antibody. Goat anti-rabbit IgG conjugated to peroxidase was used to amplify the signals, and the reacting bands were visualized by using enhanced chemiluminescence reagents. The position of Omb is shown by an arrow. Numbers between the panels represent size standards in $\mathrm{kDa}$.

\section{Identification of the Omb protein as a ferrous iron-binding protein of SC}

Since Omb shared striking homology to Eb1722 (classified as an OMR family protein), and since several OMR proteins have been characterized as ferrichrome-iron receptors (Zhai \& Saier, 2002), we suspected that Omb was also an ironbinding protein. To investigate this, we carried out fluorescence studies on purified recombinant Omb protein using the fluorescent iron indicator PG SK. The results showed that free PG SK in a simple buffered solution had an excitation maximum at $507 \mathrm{~nm}$ and an emission maximum at $532 \mathrm{~nm}$. Addition of ferrous iron or ferric iron causes quenching of PG SK fluorescence. However, the addition of Omb with iron (ferrous iron or ferric iron) markedly decreased PG SK fluorescence quenching. Neither addition of purified Omb nor addition of iron (ferrous iron or ferric iron) led to a shift of the excitation or emission spectra. Loading PG SK to a final concentration of $4 \mu \mathrm{mol} \mathrm{l}^{-1}$ to a simple buffered solution containing $10^{-6} \mathrm{M}$ ferrous iron with various concentrations of Omb $\left(10^{-6}-10^{0} \mu \mathrm{g} \mathrm{ml}^{-1}\right)$ led to a rapid increase in fluorescence in a manner dependent upon the Omb protein dose (Table 3). The more Omb present, the stronger the fluorescence. However, this phenomenon was not observed when ferrous iron was replaced with ferric iron under the same conditions as described above. This suggests that the fluorescence of PG SK was already partly quenched by ferrous iron chelation under control conditions and that redistribution of ferrous iron from the fluorescence indicator to a chelator-like Omb abolished this quenching, i.e. 'dequenched' the fluorescence. These results indicated that the Omb protein specifically binds ferrous iron with high affinity, at least in vitro.

\section{Gene expression and localization of $\mathbf{O m b}$ in $\mathbf{S 2 8 0}$}

To investigate whether Omb could or could not be produced under iron-rich conditions, and to determine the cellular location of Omb, S280 cells were grown to exponential phase in LB medium, pelleted and fractionated, and then the production and distribution of Omb in each cellular fraction was analysed by Western blotting. The results showed that Omb could be produced in both iron-rich LB medium and minimal medium and migrated as a $\sim 25 \mathrm{kDa}$ polypeptide, which agreed well with the mass predicted from the putative amino acid sequence (Fig. 2a). In addition, Omb was found only in the outer membrane fraction (Fig. 2b). Most high-affinity iron acquisition systems are not expressed constitutively and their expression is upregulated in response to a decrease in the level of intracellular iron. To investigate the effect of iron on the

Table 3. Responsiveness of PG SK to various concentrations of $\mathrm{Omb}$ with $\mathrm{Fe}^{2+}$ and $\mathrm{Fe}^{3+}$

Shown is the effect on PG SK $\left(4 \mu \mathrm{mol} \mathrm{l}^{-1}\right)$ fluorescence of various concentrations of $\mathrm{Omb}$ with $\mathrm{Fe}^{2+}$ and $\mathrm{Fe}^{3+}$ in a simple buffered solution, as recorded with a spectrofluorometer. The baseline fluorescence (excitation, $507 \mathrm{~nm}$; emission, $532 \mathrm{~nm}$ ) was recorded over $20 \mathrm{~s}$. The relative emission intensities shown are expressed as a percentage of the baseline fluorescence intensity (set at $100 \%$ ). Values shown represent mean \pm SD of three experiments.

\begin{tabular}{|c|c|c|}
\hline \multirow[t]{2}{*}{$\begin{array}{l}\text { Omb concentration } \\
\left(\mu \mathrm{g} \mathrm{ml}^{-1}\right)\end{array}$} & \multicolumn{2}{|c|}{$\begin{array}{c}\text { PG SK fluorescence } \\
\text { (percentage of baseline fluorescence) }\end{array}$} \\
\hline & $\mathrm{Fe}^{2+}\left(10^{-6} \mathrm{M}\right)$ & $\mathrm{Fe}^{3+}\left(10^{-6} \mathrm{M}\right)$ \\
\hline 0 & $6.6 \pm 0.3$ & $47.2 \pm 2.1$ \\
\hline $10^{-6}$ & $9.7 \pm 0.5$ & $47.2 \pm 2.2$ \\
\hline $10^{-5}$ & $18.4 \pm 0.4$ & $47.4 \pm 1.8$ \\
\hline $10^{-4}$ & $27.3 \pm 0.5$ & $46.5 \pm 2.3$ \\
\hline $10^{-3}$ & $41.5 \pm 0.9$ & $47.2 \pm 1.6$ \\
\hline $10^{-2}$ & $56.8 \pm 0.7$ & $47.1 \pm 1.3$ \\
\hline $10^{-1}$ & $67.2 \pm 0.8$ & $47.1 \pm 1.4$ \\
\hline 1 & $79.3 \pm 0.9$ & $47.3 \pm 1.2$ \\
\hline
\end{tabular}


expression of Omb of SC, we performed RT-PCR and Western blot analyses under various culture conditions of iron restriction. The results from RT-PCR experiments indicated that the transcription levels of omb did not increase in response to a decrease in the level of iron (data not shown). Western blot analyses confirmed the results of the RT-PCR experiments, and indicated that the Omb protein level did not increase in response to a decrease in the level of iron, even at higher concentrations of the iron chelator 2,2-DPD $\left(5 \times 10^{-4}\right.$ and $\left.1 \times 10^{-3} \mu \mathrm{M}\right)$ (data not shown). These results indicate that in SC, Omb protein is located in the outer membrane, and that Omb expression is probably not regulated by the prevailing level of iron in the environment.

\section{The absence of Omb renders SC sensitive to killing by hydrophobic antibiotics}

To demonstrate that the observed AP-sensitive and hydrophobic antibiotic-sensitive phenotype was reproducibly linked to the transposon insertion at the omb locus, we reconstructed an omb isogenic mutant, S280-2. Inactivation of the WT $o m b$ with the insertional disruption of the omb gene in S280-2 was checked by PCR, using a pair of primers complementary to sequences located in the $o m b$ and ampicillin-resistance genes (data not shown). For confirmation of the lack of Omb protein in the mutant strain S280-2, Western blot analysis of the WT and mutant S280-2 strains was performed. The results of the Western blot analysis demonstrated that the S280-2 mutant does not produce any anti-Omb-reactive protein (Fig. 2a). The isogenic mutant S280-2 was examined for sensitivity to polymyxin B and HNP-1 in suspension at different concentrations. As shown in Table 2, the mutant strain S280-2 exhibited clearly decreased MICs for polymyxin B and HNP-1 compared with the WT S280 strain. Similarly, S280-2 showed significantly enhanced susceptibilities to erythromycin and novobiocin compared with the WT strain (Table 2). These increased sensitivities to polymyxin B, HNP-1, erythromycin and novobiocin are not significantly different from those of the Tn5 mutant S280-1. Furthermore, like the Tn5 mutant S280-1, sensitivity of S280-2 to other antibiotics, including streptomycin, tetracycline, ampicillin, nalidixic acid and chloramphenicol, remained unchanged.

\section{The absence of Omb affects surface charge}

Since the increased sensitivities of the Tn5 S280-1 and S280-2 mutants seemed to be restricted to cationic APs and hydrophobic antibiotics, and since the outer membrane protein $\mathrm{Omb}$ was identified as a ferrous iron-binding protein, we suspected that the lack of Omb caused alterations in the bacterial surface charge. To investigate this, the capacity of the WT and S280-2 mutant cells to bind a highly positively charged molecule, cytochrome $c$, was determined. As shown in Fig. 3, the mutant bound a higher amount of cytochrome $c$ than the WT strain,

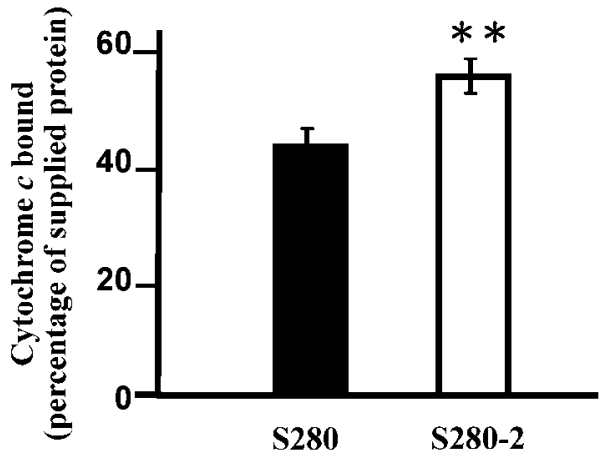

Fig. 3. Binding of the cationic protein cytochrome $c$ to WT and S280-2 mutant strains. Samples were run in triplicate and the mean and SD values for protein bound by the bacterium from three independent experiments are shown ${ }^{* *}, P<0.005$, two-tailed $t$ test).

suggesting that the cell surface is more highly negatively charged in the mutant than in the WT strain.

\section{The absence of Omb leads to attenuated growth in iron-limiting conditions}

We further examined the growth of the WT strain and mutant strain S280-2 in media under different ironlimiting conditions. The results showed that both strains grew in minimal medium containing less than $5 \times 10^{-4} \mu \mathrm{M}$ of the iron chelator 2,2-DPD, and both grew with approximately identical growth rates (Fig. 4), indicating that the ability of the mutant to utilize available iron is similar to that of the WT strain with higher levels of readily available iron. However, in minimal medium containing $5 \times 10^{-4}$ and $1 \times 10^{-3} \mu \mathrm{M}$ 2,2-DPD, which represents lower and the lowest levels of readily available iron tested, respectively, the mutant showed attenuated growth compared with the WT strain and the doubling times for the mutant were 36 and $280 \mathrm{~min}$, respectively, whereas those for the WT strain were 18 and $190 \mathrm{~min}$, respectively. The results revealed that the mutant showed attenuated growth in readily available iron-limiting conditions compared to the WT strain (Fig. 4).

\section{Complementation of the S280-2 mutant}

To confirm that (a) the attenuated growth of the S280-2 mutant in response to $5 \times 10^{-4}$ or $1 \times 10^{-3} \mu \mathrm{M} 2,2$-DPD, and (b) the AP- and hydrophobic antibiotic-sensitive phenotype of this mutant were indeed due to inactivation of the omb gene, we cloned the WT gene into plasmid pBR322; this construct (pSC2) was transformed into the S280-2 mutant strain. The results revealed that complementation of the S280-2 mutant with pSC2, but not introduction of a control plasmid, rescued the ability to exhibit WT growth rates in minimal medium containing $5 \times 10^{-4}$ or $1 \times 10^{-3} \mu \mathrm{M} 2,2$-DPD. Sensitivity measure- 


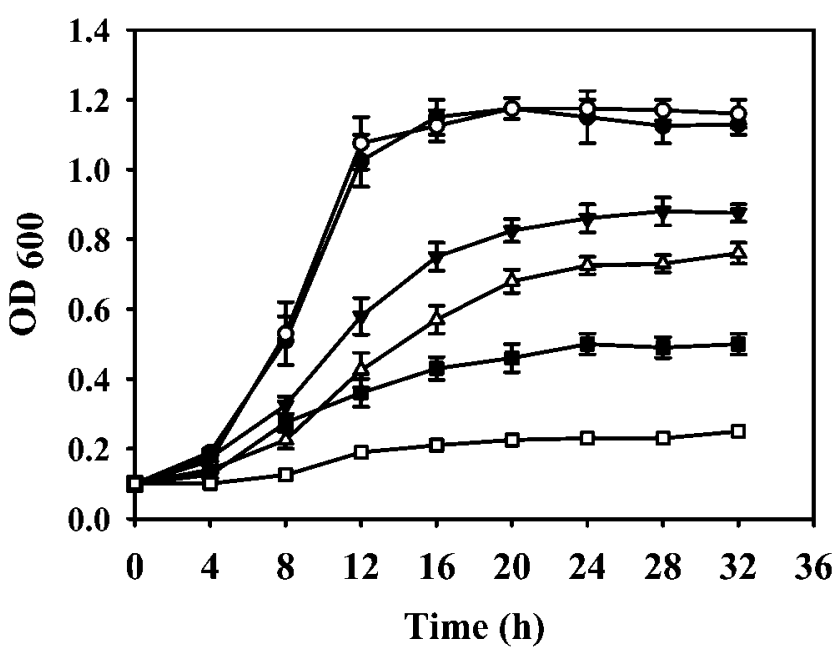

Fig. 4. Effect of ferrous iron concentration on growth of the WT S280 $(\bullet, \nabla, \mathbf{\square})$ and the mutant S280-2 $(\bigcirc, \triangle, \square)$. Cells were incubated at $37{ }^{\circ} \mathrm{C}$ in minimal medium containing the iron chelator 2,2-DPD at $0 \mu \mathrm{M}(\bullet, \bigcirc), 5 \times 10^{-4} \mu \mathrm{M}(\boldsymbol{\nabla}, \triangle)$ or $1 \times 10^{-3} \mu \mathrm{M}(\boldsymbol{\square}$, $\square$ ); the values presented are mean and SE (not shown where smaller than the symbols) of three independent experiments.

ments toward APs or hydrophobic antibiotics showed that the S280-2 mutant, when complemented with pSC2, exhibited approximately the same levels of resistance to polymyxin B, HNP-1, erythromycin and novobiocin as the WT strain (Table 2).

\section{Reduced virulence is associated with Omb}

The virulence of the isogenic mutant S280-2 and the WT strain S280 was studied by infecting BALB/c mice via either the oral or i.p. route. As shown in Table 4 , the $\mathrm{LD}_{50}$ values for mutant strain S280-2 in mice were $4.0 \times 10^{5}$ and $3.5 \times 10^{2}$ at 3 weeks post-infection via the oral and i.p. routes, respectively. These values were 20 - and 15 -fold higher than the $\mathrm{LD}_{50}$ values of $1.7 \times 10^{4}$ and $2.0 \times 10^{1}$ for WT infection in mice, administered via the oral and i.p. routes, respectively.

Table 4. Virulence of SC strains in mice

\begin{tabular}{|lcc|}
\hline $\begin{array}{l}\text { Experiment number } \\
\text { and strain }\end{array}$ & $\begin{array}{c}\mathbf{L D}_{\mathbf{5 0}} \text { (c.f.u. per mouse) for mice } \\
\text { infected by indicated route }\end{array}$ \\
\cline { 2 - 3 } & i.p. & Oral \\
\hline Experiment 1 & $2.0 \times 10^{1}$ & $1.7 \times 10^{4}$ \\
WT & $3.5 \times 10^{2}$ & $4.0 \times 10^{5}$ \\
S280-2 & & \\
Experiment 2 & $1.9 \times 10^{1}$ & $2.2 \times 10^{4}$ \\
WT & $2.8 \times 10^{2}$ & $5.4 \times 10^{5}$ \\
S280-2 & & \\
\hline
\end{tabular}

\section{DISCUSSION}

The outer membrane of enteric bacteria functions as an effective permeability barrier to harmful hydrophobic molecules. Consequently, OM impermeability is generally considered to be a characteristic feature of enteropathogenic bacteria. In this report, we have identified an outer membrane protein $(\mathrm{Omb})$ in $\mathrm{SC}$ that possesses a ferrous iron-binding capacity. We have demonstrated that the SC omb mutant is more sensitive to polymyxin B, HNP-1, erythromycin and novobiocin than the WT strain, suggesting that $\mathrm{Omb}$ is responsible for the $\mathrm{SC}$ resistance to multiple hydrophobic antimicrobial agents. Furthermore, compared with the WT strain, the SC omb mutant also showed attenuated growth in iron-limiting medium and in infection experiments. We demonstrated virulence attenuation when this mutant was administered orally or intraperitoneally to mice. Thus, Omb represents a potential virulence factor that could contribute in multiple ways to the pathogenesis of SC infection.

Although bioinformatics analysis using BLAST revealed that the putative amino acid sequence of Omb shares high degrees of identity $(>54 \%)$ with hypothetical extracytoplasmic proteins of various Gram-negative bacteria, nearly all of them are enteropathogenic bacteria. None of these Omb high-identity proteins has been characterized, except for Eb1722. In addition, because Eb1722 is classified as an OMR family protein according to bioinformatics, although without any experimental basis, the biological roles of these Omb-high-identity proteins in Gram-negative bacteria are unclear. Since we determined the location of Omb to be the SC outer membrane and demonstrated that purified recombinant $\mathrm{Omb}$ protein possessed a ferrous ironbinding ability, it is reasonable to propose that Omb may be part of an iron transport system upon which SC depends to scavenge ferrous iron from the environment. Nevertheless, Omb iron acquisition differs markedly from iron transport systems in Gram-negative bacteria that employ iron-regulated outer membrane receptors. Most Gram-negative bacteria have a Fur-like iron acquisition system whose related genes form an operon with a Fur box sequence that binds regulatory Fur repressor protein under iron-limiting conditions (Clarke et al., 2001; Wandersman \& Delepelaire, 2004). However, no Fur box sequence was found upstream of $o m b$. In addition, ambient ORFs were heterologously positioned downstream or upstream of $o m b$, indicating that the omb gene consists of a single transcriptional unit and does not form an operon with other genes. Most importantly, no change in omb expression was observed for the SC strain grown under iron-rich or different iron-restricted conditions, indicating that the expression of omb is not regulated by iron. Thus, the Omb protein may represent a novel ferrous-iron transport system that promotes the acquisition of ferrous iron by SC from the environment.

Resistance to the bactericidal effect of APs is an important property of pathogenic bacteria. Most pathogenic bacteria 
have developed a variety of strategies to combat APs. These strategies include increasing the amount of capsule polysaccharide to act as a shield against APs (Campos et al., 2004), downregulation of bactericidal peptides by bacterial plasmid DNA (Islam et al., 2001), expelling APs through energy-dependent pumps (Bayer et al., 2006; Tzeng et al., 2005), decreased fluidity of the outer membrane outer leaflet through increased acylation of lipid A (Guo et al., 1998), cleaving APs with proteases (Nyberg et al., 2004), and the repulsion of APs by reducing the net negative charge of the bacterial cell envelope through covalent modification of anionic molecules. In this study, we found that $\mathrm{Omb}$ is a ferrous iron-binding protein and that the omb mutant is more sensitive to polymyxin B and HNP-1 than the WT strain. Because of the cationic nature of polymyxin B and HNP-1, the electrostatic attraction of these two APs to the negatively charged bacterial membranes prior to membrane insertion is likely to be critical for bacterial killing (Hancock \& Chapple, 1999). In addition, the omb mutant showed significantly enhanced susceptibility to hydrophobic antibiotics (including erythromycin and novobiocin) when compared with the WT strain. Furthermore, sensitivity toward neutral antibiotics (including streptomycin, tetracycline, nalidixic acid and chloramphenicol) was the same for both the omb and WT strains. Because of the two factors above, we propose that the constitutive uptake of ferrous iron from the environment by the Omb outer membrane protein increases the positive charge at the bacterial surface sufficiently to decrease the affinity of APs and hydrophobic antibiotics for their cell wall targets, thereby affording the organism relative protection. Our finding that the omb mutant bound higher amounts of positively charged cytochrome $c$ than the WT strain is consistent with this notion. Furthermore, it has been reported that polyanionic LPS molecules of Gram-negative bacteria are electrostatically linked by divalent cations (e.g. $\mathrm{Mg}^{2+}$ and $\mathrm{Ca}^{2+}$ ) and form a 'tiled-roof structure that functions as an effective permeability barrier against hydrophobic antibiotics, detergents, dyes and macromolecules [e.g. EDTA, polymyxin B nonapeptide (PMBN), lysine polymers and protamine] (Vaara, 1992). It may be reasoned that the binding of ferrous iron by outer membrane Omb protein molecules not only reduces the negative charge of the cell envelope, but also stabilizes and maintains the integrity of the outer lipopolysaccharide layer and cell wall, and thereby enhances the barrier.

The role of iron, as a regulator for virulence expression, was first established by Pappenheimer \& Johnson (1936). Alteration of available iron levels, brought about by inherited disease or tissue injury, predisposes humans to infection with a variety of pathogens (Bullen et al., 2006). However, the level of free iron in biological fluids of healthy humans is very tightly limited and controlled (Weinberg, 1978). To obtain this unavailable iron, most pathogenic bacteria including Salmonella spp. have to lyse host cells or induce expression of haemophores and siderophores via the iron-responsive Fur regulon (Clarke et al., 2001; Wandersman \& Delepelaire, 2004). We propose that the availability of iron via these routes is insufficient to meet the needs of SC in the early stages of infection. In this study, we identified a novel protein, Omb, in the SC outer membrane with ferrous iron-binding ability that may deliver sufficient additional iron to the bacterium. Since the results of the fluorescence analysis indicated that purified recombinant Omb is a high-affinity ferrous iron-binding protein, and since the expression of $\mathrm{Omb}$ in SC is independent of iron supply and appears to be constitutive, it would be reasonable to speculate that ferrous iron uptake by $\mathrm{Omb}$ in SC is unique and very important for early stage infection before other iron acquisition systems become induced. Bacterial pathogens contain several iron acquisition systems, so that mutation of one system can be compensated for by another system, thus protecting the organism from virulence attenuation (Nassif et al., 1987; Wu et al., 2002; Wyckoff et al., 2006). In contrast, the virulence of our constructed omb isogenic mutant became attenuated in mice compared with the WT strain, which is consistent with the notion that Omb in SC is unique and very important for early stage infection. Although systems involved in ferrous iron uptake and usage in SC are beginning to be investigated, the precise mechanism by which these bacteria are able to bind and transport ferrous iron through their membranes is not well understood.

In summary, these data represent the first description, to our knowledge, of Omb as a novel outer membrane protein that is very important for scavenging ferrous iron. It is possible that the absence of Omb causes structural changes, with increased net negative charge on the surface, thereby facilitating interaction of APs and hydrophobic antibiotics with the bacterial surface. The BLAST search revealed that at least 20 different bacterial species contain a putative Omb protein $(>54 \%$ identity), with only one such protein found in each of these species. Since all of these species are Gram-negative enteropathogenic bacteria, it would be interesting to investigate whether Omb is also a ferrous iron-binding protein involved in hydrophobic antibiotic resistance in any of these other bacteria. Such studies may provide a strategy for designing new drugs or novel combinations of drugs for the treatment of Gram-negative enteropathogenic infections including those with SC.

\section{ACKNOWLEDGEMENTS}

This work was supported by a grant (97-EC-17-A-10-S1-0013) from the Department of Industrial Technology, Ministry of Economic Affairs of Taiwan.

\section{REFERENCES}

Bayer, A. S., Kupferwasser, L. I., Brown, M. H., Skurray, R. A., Grkovic, S., Jones, T., Mukhopadhay, K. \& Yeaman, M. R. (2006). Low-level resistance of Staphylococcus aureus to thrombin-induced 
platelet microbicidal protein 1 in vitro associated with qacA gene carriage is independent of multidrug efflux pump activity. Antimicrob Agents Chemother 50, 2448-2454.

Beliavskaia, V. A., Timofeev, I. V., Perminova, N. G., Paletskaia, T. F., Kozhina, E. M. \& Zagrebel'nyi, S. N. (2000). Construction of an expression plasmid vector for accomplishing in vivo delivery of recombinant biologically active proteins. 2. Synthesis of HBcAG in a vaccine strain of Salmonella choleraesuis. Mol Gen Mikrobiol Virusol 3, 17-21.

Bullen, J. J., Rogers, H. J., Spalding, P. B. \& Ward, C. G. (2006), Natural resistance, iron and infection: a challenge for clinical medicine. J Med Microbiol 55, 251-258.

Campos, M. A., Vargas, M. A., Regueiro, V., Llompart, C. M., Alberti, S. \& Bengoechea, J. A. (2004). Capsule polysaccharide mediates bacterial resistance to antimicrobial peptides. Infect Immun 72, 7107-7114.

Chang, M. C., Chang, J. C. \& Chen, J. P. (1993). Cloning and nucleotide sequence of an extracellular $\alpha$-amylase gene from Aeromonas hydrophila MCC-1. J Gen Microbiol 139, 3215-3223.

Chiu, C. H., Tang, P., Chu, C., Hu, S., Bao, O., Yu, J., Chou, Y. Y., Wang, H. S. \& Lee, Y. S. (2005). The genome sequence of Salmonella enterica serovar Choleraesuis, a highly invasive and resistant zoonotic pathogen. Nucleic Acids Res 33, 1690-1698.

Chiu, C. H., Chuang, C. H., Chiu, S., Su, L. H. \& Lin, T. Y. (2006), Salmonella enterica serotype Choleraesuis infections in pediatric patients. Pediatrics 117, e1193-e1196.

Clarke, T. E., Tari, L. W. \& Vogel, H. J. (2001). Structural biology of bacterial iron uptake systems. Curr Top Med Chem 1, 7-30.

Cornelis, P., Digneffe, C. \& Willemot, K. (1982). Cloning and expression of a Bacillus coagulans amylase gene in Escherichia coli. Mol Gen Genet 186, 507-511.

Daldal, F. (1984). Nucleotide sequence of gene $p f k B$ encoding the minor phosphofructokinase of Escherichia coli K-12. Gene 28, 337-342.

Doig, P. \& Trust, T. J. (1994). Identification of surface-exposed outer membrane antigens of Helicobacter pylori. Infect Immun 62, 4526-4533.

Donnenberg, M. S. \& Kaper, J. B. (1991). Construction of an eae deletion mutant of enteropathogenic Escherichia coli by using a positive-selection suicide vector. Infect Immun 59, 4310-4317.

Guo, L., Lim, K. B., Poduje, C. M., Daniel, M., Gunn, J. S., Hackett, M. \& Miller, S. I. (1998). Lipid A acylation and bacterial resistance against vertebrate antimicrobial peptides. Cell 95, 189-198.

Hancock, R. E. W. \& Chapple, D. S. (1999). Peptide antibiotics. Antimicrob Agents Chemother 43, 1317-1323.

Hensel, M., Shea, J. E., Gleeson, C., Jones, M. D., Dalton, E. \& Holden, D. W. (1995). Simultaneous identification of bacterial virulence genes by negative selection. Science 269, 400-403.

Islam, D., Bandholtz, L., Nilsson, J., Wigzell, H., Christensson, B., Agerberth, B. \& Gudmundsson, G. H. (2001). Downregulation of bactericidal peptides in enteric infections: a novel immune escape mechanism with bacterial DNA as a potential regulator. Nat Med 7, 180-185.

Knight, S. A. B., Vilaire, G., Lesuisse, E. \& Dancis, A. (2005). Iron acquisition from transferrin by Candida albicans depends on the reductive pathway. Infect Immun 73, 5482-5492.

Kristian, S. A., Dürr, M., Van Strijp, J. A., Neumeister, B. \& Peschel, A. (2003). MprF-mediated lysinylation of phospholipids in Staphylococcus aureus leads to protection against oxygen-independent neutrophil killing. Infect Immun 71, 546-549.

Kristian, S. A., Datta, V., Weidenmaier, C., Kansal, R., Fedtke, I., Peschel, A., Gallo, R. L. \& Nizet, V. (2005). D-Alanylation of teichoic acids promotes group A Streptococcus antimicrobial peptide resistance, neutrophil survival, and epithelial cell invasion. J Bacteriol 187, 6719-6725.

Mildiner-Earley, S. \& Miller, V. L. (2006). Characterization of a novel porin involved in systemic Yersinia enterocolitica infection. Infect Immun 74, 4361-4365.

Nassif, X., Mazert, M. C., Mounier, J. \& Sansonetti, P. J. (1987). Evaluation with an iuc:Tn10 mutant of the role of aerobactin production in the virulence of Shigella flexneri. Infect Immun 55, 1963-1969.

Nyberg, P., Rasmussen, M. \& Bjorck, L. (2004). $\alpha_{2}$-Macroglobulinproteinase complexes protect Streptococcus pyogenes from killing by the antimicrobial peptide LL-37. J Biol Chem 279, 52820-52823.

Pappenheimer, A. M., Jr \& Johnson, S. J. (1936). Studies in diphtheria toxin production. I. The effect of iron and copper. Br J Exp Pathol 17, 335-341.

Petrat, F., Rauen, U. \& de Groot, H. (1999). Determination of the chelatable iron pool of isolated rat hepatocytes by digital fluorescence microscopy using the fluorescent probe, phen green SK. Hepatology 29, 1171-1179.

Reed, L. J. \& Muench, H. (1938). A simple method of estimating the fifty percent endpoints. Am J Hyg 27, 493-497.

Sambrook, J., Fritsch, E. F. \& Maniatis, T. (1989). Molecular Cloning: a Laboratory Manual, 2nd edn. Cold Spring Harbor, NY: Cold Spring Harbor Laboratory.

Schwartz, K. J. (1999). Salmonellosis. In Diseases of Swine, 8th edn, pp. 535-551. Edited by B. E. Straw, S. D’Allaire, W. L. Mengeling \& D. J. Taylor. Ames, IA: Iowa State University.

Tamayo, R., Choudhury, B., Septer, A., Merighi, M., Carlson, R. \& Gunn, J. S. (2005). Identification of $c p t A$, a PmrA-regulated locus required for phosphoethanolamine modification of the Salmonella enterica serovar Typhimurium lipopolysaccharide core. J Bacteriol 187, 3391-3399.

Tzeng, Y. L., Ambrose, K. D., Zughaier, S., Zhou, X., Miller, Y. K., Shafer, W. M. \& Stephens, D. S. (2005). Cationic antimicrobial peptide resistance in Neisseria meningitidis. J Bacteriol 187, 53875396.

Vaara, M. (1992). Agents that increase the permeability of the outer membrane. Microbiol Rev 56, 395-411.

von Heijne, G. (1983). Patterns of amino acids near signal-sequence cleavage sites. Eur J Biochem 133, 17-21.

Wandersman, C. \& Delepelaire, P. (2004). Bacterial iron sources: from siderophores to hemophores. Annu Rev Microbiol 58, 611-647.

Wang, J. Y., Hwang, J. J., Hsu, C. N., Lin, L. C. \& Hsueh, P. R. (2006). Bacteraemia due to ciprofloxacin-resistant Salmonella enterica serotype Choleraesuis in adult patients at a university hospital in Taiwan. Epidemiol Infect 134, 977-984.

Weinberg, E. D. (1978). Iron and infection. Microbiol Rev 42, 45-66.

Wu, W. S., Hsieh, P. C., Huang, T. M., Chang, Y. F. \& Chang, C. F. (2002). Cloning and characterization of an iron regulated locus, IroA, in Salmonella enterica serovar Choleraesuis. DNA Seq 13, 333-341.

Wyckoff, E. E., Mev, A. R., Leimbach, A., Fisher, C. F. \& Payne, S. M. (2006). Characterization of ferric and ferrous iron transport systems in Vibrio cholerae. J Bacteriol 188, 6515-6523.

Zhai, Y. \& Saier, M. H. (2002). The beta-barrel finder (BBF) program, allowing identification of outer membrane beta-barrel proteins encoded within prokaryotic genomes. Protein Sci 11, 2196-2207.

Edited by: A. R. Walmsley 